\section{Informationsmanagement einer Landschaft im Wandel}

\author{
von Ronny Weinkauf, SCC Software GmbH \\ Merseburg, und Renate Patz, Fachhoch- \\ schule Merseburg und Förderverein Zent- \\ ralwerkstatt Pfännerhall e.V.
}

Landschaften resp. Regionen, in denen sich ein komplexer Wandel vollzieht, stellen für die involvierten Akteure eine neue Herausforderung dar, um im Wettbewerb der Regionen, auch im Wettbewerb der Standorte bestehen zu können. Ein grundsätzliches Problem besteht darin, die Vielzahl an Informationsinseln einer Region, Informationsangebote wie Informationsanbieter so zu vernetzen, dass nicht lediglich nur eine neue Informationssammlung entsteht, sondern dass Informationsmanagement als Prozess und kooperative Entwicklungsstrategie aufgefasst und gestaltet wird. Für die Bergbaufolgelandschaft Geiseltal wurde mit Mitteln des Landes Sachsen-Anhalt ein Konzept erarbeitet, mit dessen Umsetzung vor wenigen Monaten begonnen wurde.

\section{Motivation und Hintergrund}

Räumliche Strukturen, Nutzungen, Funktionen, Potenziale einer Landschaft ändern sich naturwie anthrogenbedingt stetig. Zielorientierte Landschaftsentwicklung und Landnutzung erfordern folglich eine gesicherte Informationsbasis über den betreffenden Raum, seiner Elemente und deren funktionalen Zusammenhänge. Die Zielorientierung Nachhaltigkeit setzt geeignete Bewertungsmaßstäbe für eine transparente Auswahl von Empfehlungen oder Angeboten voraus.

Für Regionen, in denen sich tief greifende Wandlungsprozesse vollziehen, ist es von eminenter Bedeutung, die Veränderungen, den damit einhergehenden Identitäts- und Imagewandel, die entstehenden neuen Angebote nach außen und auch innerhalb der Region publik zu machen. Die Erarbeitung einer schlüssigen Gesamtstrategie, eines stimmigen Gesamtbildes, die Kommunikation der regionalen Akteure untereinander und mit weiteren überregionalen Akteuren, deren Kooperation kann durch ein adäquates Informationsmanagement befördert werden. Letztlich sind nutzerfreundliche
Informationsdienstleistungen für die Zufriedenheit der Gäste einer Region oder potenzieller Investoren unerlässlich. Betrachtet werden hier ausschließlich rechnerbasierte Informationssysteme.

Kein Einzelfall ist die nachfolgend geschilderte Situation, entsprechende Analysen und Erhebungen, auch eigene im betrachteten Untersuchungsraum (Patz et al. 2003), bestätigen dies:

Das Konglomerat an Informationen über eine Region sowie an Informationsanbietern wuchert permanent. Informationen $\mathrm{zu}$ einer Region liegen in unterschiedlichen Medien vor, digitale Informationen sind unterschiedlich strukturiert, inhaltlich nicht immer konsistent, sie nehmen nur bedingt aufeinander Bezug. Es fehlt ein Informationszugang für eine gesamte Region. Informationen werden in der Regel als Text oder Bild zur Verfügung gestellt, allerdings ohne direkten Raumbezug, das Wieder finden in einer Landkarte wird nicht adäquat unterstützt, was allerdings Geographische Informationssysteme voraussetzt. Besondere Schwächen zeigen sich meist bei den dynamischen Inhalten von Web-Seiten. Regionale Akteure verfügen nur partiell über die informationstechnische oder gestalterische Kompetenz, die infrastrukturellen Voraussetzungen sind lückenhaft. Auch wenn es gute „Insellösungen“ gibt, so müssen diese integriert, besser verwaltet und gepflegt, in überregionale Informationssysteme eingebunden werden. Dem an Informationen interessierten Nutzer wird es also oftmals nicht leicht gemacht, sich in der Informationsvielfalt zurechtzufinden. Die Bewertung der Empfehlungen oder Angebote wird dem Nachfragenden überlassen.

\section{Die Spezifika von Bergbaufolgeland- schaften. Das Beispiel Geiseltal}

Zugespitzt verläuft der Prozess des Wandels von Landschaften in den ehemaligen ostdeutschen Bergbauregionen wie oben skizziert. Es sind Übergangslandschaften, deren stabiler Endzustand erst nach Jahren bzw. Jahrzehnten erreicht sein wird. Das Geiseltal ist eine Region in Mitteldeutschland, geprägt von einem 300jährigen Braunkohleabbau sowie dessen Folgen, nunmehr hinstrebend zu einer Seenlandschaft mit starkem Akzent auf Erholungs-, Freizeit- und Tourismusnutzungen. Seit Juni 2003 wird das 
letzte und größte der drei Tagebaurestlöcher geflutet, in wenigen Jahren wird der entstehende Geiseltalsee mit 19 km2 Wasserfläche das größte künstliche Gewässer Deutschlands sein (LMBV 2003). Die Herausforderungen an die nachhaltige Planung und Gestaltung einer solcher Landschaft sind immens, es sind sowohl die Ansiedlung, die Erhaltung und der Schutz raumspezifisch vorhandenen Naturkapitals als auch eine angemessene wirtschaftliche und sozial gesicherte Entwicklung zu berücksichtigen.

Auch wenn neue Seen entstehen, nicht nur im Geiseltal, ebenso im Bitterfelder Raum, im Südraum Leipzig und in der Lausitz, klassische Tourismusregionen mit sicht- und erlebbaren Angebotselementen in genügender Zahl sind es noch nicht. Will man im Wettbewerb zwischen diesen Regionen aber auch mit anderen bereits etablierten Angeboten erfolgreich bestehen, so sind Marketing und Informationsstrategie aufeinander abzustimmen (Luft 2001). Zentrale Anlauf- und Informationspunkte bzw. City- oder Regionalmanager übernehmen dabei wichtige Funktionen. Für das Besucherziel Geiseltal wurde daraus zunächst die Forderung nach einem Management der relevanten Informationsbestände abgeleitet, welches die Erfassung, Pflege und Bereitstellung der Informationen organisiert. Dies war das Ziel eines vom Land Sachsen-Anhalt geförderten Projektes (Laufzeit Mai bis Dezember 2002) zur Erarbeitung eines entsprechenden Konzeptes durch den Förderverein „Zentrum für Zukunftstechnologie, Kunst und Design. Zentralwerkstatt Pfännerhall. Geiseltal“ e.V.. In die Projektbearbeitung wurden die Kompetenzen der Fachhochschule Merseburg und der SCC Software GmbH einbezogen.

\section{Ist-Analyse. Ableitung der organisatori- schen und informationstechnischen An- forderungen}

Ausgangspunkt war eine Erhebung zur Erfassung des Ist-Zustandes der Informationsverarbeitung im Geiseltal. Mittels Interview, Beobachtung und Dokumentenanalyse wurden die existierenden Informationssysteme, die Informationsanbieter und deren Informationsangebote sowie Informationsbedarfe und -nachfragen ermittelt. Das Bild der Informationslandschaft im Geiseltal spiegelt sich in der obigen Situationsschilderung wider. Deutlich wurde bei den Er- hebungen: Informationsmanagement ist ein äußerst sensibler Prozess, beginnend bereits bei der Auswahl der einzubeziehenden Akteure.

Zur Abgrenzung des Diskursbereichs wurde auf ein Akteursmodell zurückgegriffen, welches auf der Grundlage empirischer Untersuchungen zur regionalen Entwicklung erstellt wurde (Henn und Patz 2003). Welche Akteure sind für das regionale Informationsmanagement relevant? Bezieht man die betriebswirtschaftliche Betrachtungsweise auf den Informationsaustausch, so können Akteure Anbieter von Informationen und/oder Nachfrager von Informationen sein. Naherholer, Touristen und Bewohner fragen primär Informationen nach, wobei insbesondere Bewohner auch zunehmend zum Anbieter von Informationen werden. Die Administrative - im Geiseltal im Wesentlichen die kommunalen Verwaltungen - hingegen tritt als Anbieter von Informationen auf, wie auch Vereine und Verbände sowie Teile der ansässigen Unternehmen.

Der Kommunikation mit den Akteuren muss ein mindest gleich großes Augenmerk gewidmet werden wie der rein informationstechnischen Seite. Ohne deren Akzeptanz und Mitwirkung kann kein funktionierendes Informationsmanagement aufgebaut werden. Dies heißt, auf typische marketing- und organisationsrelevante, informationstechnische und strukturelle Fragen und Probleme einzugehen. Dazu zählen die abgrenzbaren Zielstellungen für das Informationssystem/Informationsmanagement, die Definition der Anbietergruppen, die Definition der Nutzer- bzw. Zielgruppen und deren zielgenaues Erreichen, die eindeutige Zuordbarkeit zwischen Anbieter und Nachfrager, die Quantifizierung des Nutzens, des Mehrwertes, die Sicherung der Qualität und Zuverlässigkeit der angebotenen Informationen, die Daten- und Systemrechte, die Wahl der Informationsmedien und weiteres mehr.

Diese Überlegungen und Ergebnisse waren in dem zu entwickelnden Konzept für das Informationssystem Geiseltal zu berücksichtigen. Angesichts der zentralen Bedeutung für das Geiseltal wurde das Konzept beispielhaft auf touristische, kulturelle und fachwissenschaftliche Inhalte bezogen, um Gäste und Investoren diesbezüglich mit den gewünschten Informationen zu versorgen. 


\section{Ein Stufenkonzept als Lösungsweg}

Informationsmanagement (vgl. u. a. Heinrich 2002; Kcmar 2003) nutzt Vorgehensmodelle der Steuerung des Entwicklungsprozesses mit dem Ziel, Anwendungssysteme so wirtschaftlich wie möglich zu erstellen. Durch eine Gruppierung der Tätigkeiten nach sachlogischen oder ergebnisorientierten Gesichtspunkten wird eine bessere Handhabbarkeit erreicht. Die Gruppierungen, auch als Phasen bezeichnet, dienen dazu, den gesamten Entwicklungsprozess mit definierten Zwischenergebnissen und präzisen Prüfpunkten (Meilensteinen) in plan- und kontrollierbare Einheiten zu zerlegen. In der öffentlichen Verwaltung gelten die „Besonderen Vertragsbedingungen für die Planung von DV-gestützten Verfahren“ (Stahlknecht und Hasenkamp 2002). Es wird empfohlen, Softwarelösungen nach dem V-Modell zu implementieren (Bröhl und Dröschel 1995). Der erste Abschnitt, die Verfahrensplanung, umfasst die Phasen Verfahrensidee, Ist-Analyse, Forderungen, Grobkonzept und fachliches Feinkonzept. Da erwartet wurde, dass die öffentlichen Einrichtungen wesentlich am regionalen Informationsmanagement $\mathrm{zu}$ beteiligen sind und das bekannte Vorgehensmodell die kooperative Arbeit erleichtert, wurde dem folgend der Aufbau des Informationssystems in vier aufeinander folgenden und aufeinander aufbauenden Stufen vorgeschlagen:

\section{Stufe 1: Lokales Besucherinformationssystem}

Neben der Erarbeitung der Konzeption selbst werden thematische Karten erstellt und über eine Oberfläche für Besucherterminals zugänglich gemacht. Beispielhaft realisiert wurden interaktive Karten durch sog. Fachschalen zu den Themengebieten „Landmarken und Aussichtpunkte“ und „Wege- und Besucherlenkung“.

\section{Stufe 2: Dezentrales Informationssystem}

Den Schwerpunkt bilden regionale Informationsdienste (Webservices), welche in die bestehenden Websites eingebunden werden. Dadurch sollen insbesondere dynamische Inhalte wie Termine leichter gepflegt und bereitgestellt werden können. Mit diesen Informationen können weitere dezentrale Infoterminals dynamisch gespeist werden. Parallel wird die raumbezogene Informationsbasis erweitert, es werden zielgruppenbezogene, thematische Karten erzeugt, z. B. Karten „Sportmöglichkeiten“ oder „Gewerbeflächen“.

Stufe 3: Personalisiertes Informationssystem

Die Webangebote werden personalisiert, d. h., die Angebote auf spezielle Nutzergruppen zugeschnitten, die Inhalte erscheinen aufbereitet je nach Nutzertyp. Die unterschiedlichen Nutzergruppen - im Geiseltal-Netz ca. 6.000 Haushalte, 10 Schulen, 300 Unternehmen, 11 Kommunalverwaltungen - werden über ein regionsinternes Netz miteinander und mit dem Internet verbunden. Als Optionen werden interaktive GIS-Anwendungen, elektronische Gästekarten und der Einsatz von Webcams vorbereitet.

\section{Stufe 4: Mobiles Informationssystem}

Es erfolgt die Bereitstellung örtlich gebundener Informationen und Dienste (sog. Location based Services) bezogen auf den jeweiligen Standort des Nutzers. Beispiele sind Städteführer mit Sehenswürdigkeiten, der Darstellung ihrer Historie und des Originalzustandes, Fahrpläne von Bus und Bahn inkl. Stadtkarte, Hinweise auf besondere Örtlichkeiten, wie z. B. Parks, Telefonzellen, Toiletten, Auswahl und Bestellung aus einer Speisekarte im Restaurant. Diese Informationen erhält der Nutzer neben den Infoterminals auch über sein eigenes mobiles Endgerät (z. B. seinen Personal Digital Assistant).

\section{Stand der Realisierung und Ausblick}

Dieses vierstufige Konzept wurde mehrfach öffentlich bzw. direkt bei den Akteuren präsentiert. Dadurch wurde der Bekanntheitsgrad der Konzeption in der Region erhöht und die regionalen Akteure wurden in die Diskussion mit einbezogen. In dem derzeit laufenden Folgeprojekt, vom Land Sachsen-Anhalt als PublicPrivate-Partnership-Projekt gefördert (Laufzeit November 2003 bis Dezember 2004), wird dieses Stufenkonzept nun schrittweise realisiert. Damit entsteht eine durchgängige Informationsund Kommunikationsinfrastruktur, eine Plattform digitaler, regionaler Informationen zu verschiedenen Themen mit Geobezug für die Region Geiseltal und Umgebung, die auch über Schnittstellen in andere Informationssysteme des Landes eingebunden werden können. Neue Formen der Wissens- und Informationsvermittlung für die verschiedensten Akteure werden etabliert und ausgebaut. Durch eine wissen- 
schaftliche Begleituntersuchung, die Aufnahme des Arbeitspaketes „Diskurs“ in das laufende Vorhaben wird dem Informationsmanagement als Gestaltungsaufgabe auch weiterhin das angemessene Gewicht eingeräumt.

\section{Literatur}

Bröhl, A.-P., Dröschel, W., 1995: Das V-Modell: Der Stand für die Softwareentwicklung mit Praxisleitfaden. München: Oldenburg-Verl., 2. Aufl.

Heinrich, L., 2002: Informationsmanagement. München: Oldenburg-Verl., 7. Aufl.

Henn, A.; Patz, R.,. 2003: Qualität des Naturdargebotes in der Landnutzung. Soziale und ökonomische Bewertung als Instrument der Entscheidungsunterstützung. Technikfolgenabschätzung. Theorie und Praxis, Nr. 2, 12. Jg. Juni 2003, S. 104-110

Krcmar, H., 2003: Informationsmanagement. Berlin: Springer-Verl., 3. Aufl.

LMBV/Lausitzer- und Mitteldeutsche Bergbauverwaltungsgesellschaft, 2003: Flutung des Tagebaurestloches Mücheln. Bitterfeld: Eigenverlag

Luft, H., 2001: Organisation und Vermarktung von Tourismusorten und Tourismusregionen: Destination Management. Meßkirch: Gmeiner Verlag

Patz, R.; Weinkauf, R.; Weinkauf, A.; Rehwaldt, T., 2003: Das Geiseltal im Wandel. Inszenierungen und Diskurs des Wandels als identitätsfördernde Faktoren. Braunsbedra; Projektbericht

Stahlknecht, P.; Hasenkamp, U., 2002: Einführung in die Wirtschaftsinformatik. Berlin u.a.: SpringerVerlag, 10. Aufl.

\section{Kontakt}

Dr. Ronny Weinkauf

SCC Software GmbH

Fritz-Haber-Str. 9, 06217 Merseburg

Tel.: +49 (0) 3461 / 2599510

Fax: +49 (0) 3461 / 2599511

E-Mail: ronny.weinkauf@scc-web.de

Dr. Renate Patz

Fachhochschule Merseburg

Prorektorat für Forschung und Technologietransfer

Geusaer Str., 06217 Merseburg

Tel.: +49 (0) 3461 / 462907

Fax: +49 (0) 3461 / 462919

E-Mail: renate.patz@ltg.fh-merseburg.de 